ESAIM: PROCEEDINGS, March 2013, Vol. 39, p. 25-31

M. Belhaq, P. Lafitte and T. Lelièvre Editors

\title{
EXISTENCE AND CONVERGENCE OF AN MHD APPROXIMATE DECONVOLUTION MODEL
}

\author{
Luigi C. Berselli ${ }^{1}$, Davide Catania ${ }^{2}$ and Roger Lewandowski $^{3}$
}

Résumé. On considère un modèle pour la simulation des tourbillons à grande échelle pour les équations de la magnétohydrodynamique (MHD). On étudie un modèle alpha obtenu par la méthode de Stolz et Adams, qui utilisent des opérateurs de déconvolution à la van Cittert pour l'approximation des équations. On considère des conditions au bord périodiques et on utilise le filtre de Helmholtz. On montre l'existence et l'unicité d'une solution faible régulière pour un système avec filtre et déconvolution dans les deux équations. On montre aussi que la solution converge à la solution des équations filtrées de la MHD, au sens approprié, lorsque le paramètre de la déconvolution va à l'infini. On peut étendre ces résultats au problème avec le filtre seulment pour l'équation de la vitesse.

\begin{abstract}
We consider a Large Eddy Simulation (LES) model for the equations of Magnetohydrodynamics (MHD). We study an $\alpha$-model that is obtained by adapting to the MHD the approach by Stolz and Adams with van Cittert approximate deconvolution operators. We work with periodic boundary conditions and use the Helmholtz filter. We prove existence and uniqueness of a regular weak solution for a system with filtering and deconvolution in both equations. We show that when the deconvolution parameter goes to infinity, then the solution converges - in an appropriate sense - to the solution of the filtered MHD equations. These results can be extended to the problem with filtering acting only on the velocity.
\end{abstract}

\section{INTRODUCTION}

Let us consider the equations of (double viscous) incompressible Magnetohydrodynamics (MHD),

$$
\begin{gathered}
\partial_{t} \mathbf{u}+\nabla \cdot(\mathbf{u} \otimes \mathbf{u})-\nabla \cdot(\mathbf{B} \otimes \mathbf{B})+\nabla p=\nu \Delta \mathbf{u}+\mathbf{f}, \\
\partial_{t} \mathbf{B}+\nabla \cdot(\mathbf{B} \otimes \mathbf{u})-\nabla \cdot(\mathbf{u} \otimes \mathbf{B})=\mu \Delta \mathbf{B}, \\
\nabla \cdot \mathbf{u}=\nabla \cdot \mathbf{B}=0, \\
\mathbf{u}(0, \mathbf{x})=\mathbf{u}_{0}(\mathbf{x}), \quad \mathbf{B}(0, \mathbf{x})=\mathbf{B}_{0}(\mathbf{x}),
\end{gathered}
$$

where $\nu>0$ is the kinematic viscosity, while $\mu>0$ is the magnetic diffusivity. The field $\mathbf{u}$ is the velocity, the field $\mathbf{B}$ is the magnetic field, while the scalar $p$ is the pressure. We assume the three dimensional setting, and the technical difficulties are at least those known for the 3D Navier-Stokes equations. Examples of fluids that can be described by the equations (1) are plasmas, liquid metals, and salt water or electrolytes [8]. In particular, we want to study some turbulence MHD models, originally developed for the Navier-Stokes equations by Stolz and Adams [1,17,18].

\footnotetext{
${ }^{1}$ Dipartimento di Matematica Applicata "U. Dini”, Università di Pisa, Via F. Buonarroti 1/c, I-56127 Pisa, ITALY, berselli@dma.unipi.it, http ://users.dma.unipi.it/berselli

${ }^{2}$ Dipartimento di Matematica, Università di Brescia, Via Valotti 9, I-25133 Brescia, ITALY, davide.catania@ing.unibs.it, http ://www.ing.unibs/ davide.catania/

${ }^{3}$ IRMAR, UMR 6625, Université Rennes 1, Campus Beaulieu, 35042 Rennes cedex FRANCE,

Roger.Lewandowski@univ-rennes1.fr, http ://perso.univ-rennes1.fr/roger.lewandowski/
} 
In the recent years, the topic has attracted the interest of many researchers and, especially for the study of existence, uniqueness, regularity, and estimates on the number of degrees of freedom, we recall $[6,7,10-13,16]$.

In the context of Approximate Deconvolution Models for turbulent flows, see the recent overviews in $[14,15]$. The issue of the limiting behavior of solutions in terms of the deconvolution parameter is a very recent topic $[2,5]$. As to $\mathrm{MHD}$, the topic seems not explored yet, hence we consider here equations with the magnetic field.

To briefly introduce the problem, we recall that the main underlying idea of LES $[4,5]$ is to compute at least the "mean values" of the flow fields, which are the velocity field $\mathbf{u}=\left(u^{1}, u^{2}, u^{3}\right)$, the magnetic field $\mathbf{B}=\left(B^{1}, B^{2}, B^{3}\right)$ and the scalar pressure field $p$. This is heuristically motivated from the fact that some gross characteristics of the flow behave in a more orderly manner. In the spirit of the work started probably with Reynolds, this corresponds to find a suitable computational decomposition

$$
\mathbf{u}=\overline{\mathbf{u}}+\mathbf{u}^{\prime} \quad \mathbf{B}=\overline{\mathbf{B}}+\mathbf{B}^{\prime} \quad \text { and } \quad p=\bar{p}+p^{\prime},
$$

where the primed variables are fluctuations around the over-lined mean fields. In practical applications, knowledge of the mean flow is enough to extract relevant information on the motion.

In our context, the "mean values" are defined by applying the inverse of a differential operator. Denoting the mean fields by $\overline{\mathbf{u}}, \overline{\mathbf{B}}$ and $\bar{p}$, and by assuming that the averaging operation commutes with differential operators, one gets the filtered MHD equations

$$
\begin{gathered}
\partial_{t} \overline{\mathbf{u}}+\nabla \cdot(\overline{\mathbf{u} \otimes \mathbf{u}})-\nabla \cdot \overline{(\mathbf{B} \otimes \mathbf{B})}-\nu \Delta \overline{\mathbf{u}}+\nabla \bar{p}=\overline{\mathbf{f}} \\
\partial_{t} \overline{\mathbf{B}}+\nabla \cdot \overline{(\mathbf{B} \otimes \mathbf{u})}-\nabla \cdot \overline{(\mathbf{u} \otimes \mathbf{B})}=\mu \Delta \overline{\mathbf{B}} \\
\nabla \cdot \overline{\mathbf{u}}=\nabla \cdot \overline{\mathbf{B}}=0 \\
\overline{\mathbf{u}}(0, \mathbf{x})=\overline{\mathbf{u}_{0}}(\mathbf{x}), \quad \overline{\mathbf{B}}(0, \mathbf{x})=\overline{\mathbf{B}}_{0}(\mathbf{x}) .
\end{gathered}
$$

This raises the question of the interior closure problem, that is the modeling of the tensors

$$
\overline{(\mathbf{c} \otimes \mathbf{d})} \quad \text { with } \mathbf{c}, \mathbf{d}=\mathbf{u} \text { or } \mathbf{B}
$$

in terms of the filtered variables $(\overline{\mathbf{u}}, \overline{\mathbf{B}}, \bar{p})$. The model that we study in this paper is the Approximate Deconvolution Model (ADM), introduced by Adams and Stolz [1,17]. This model is defined by approximating the filtered bi-linear terms as follows :

$$
\overline{(\mathbf{c} \otimes \mathbf{d})} \sim \overline{\left(D_{N} \overline{\mathbf{c}} \otimes D_{N} \overline{\mathbf{d}}\right)}
$$

Here the filtering operators $G_{i}$ are defined thanks to the Helmholtz filter by $G_{1}(\mathbf{u})=\overline{\mathbf{u}}, G_{2}(\mathbf{B})=\overline{\mathbf{B}}$, where in the paper $G_{i}:=\left(\mathrm{I}-\alpha_{i}^{2} \Delta\right)^{-1}, i=1,2$. Let us observe that we can then have two different filters corresponding to the equation for the velocity and for the magnetic field. There are two interesting values for the couple $\left(\alpha_{1}, \alpha_{2}\right) \in \mathbb{R}^{+} \times \mathbb{R}^{+}$.

(1) $\alpha_{1}=\alpha_{2}>0$ : in this case the approximate equations conserve Alfvén waves [12].

(2) $\alpha_{1}>0, \alpha_{2}=0$, which means no filtering in the equation for $\mathbf{B}$; this is possible since the equation for the magnetic field turns out to behave much better than that for the velocity.

For simplicity, we will present here just the first case with double filtering (see [3] for the other case). In order to handle better the approach with two filters, we will use the notation associated to the operators $G_{i}$. Consequently, the deconvolution operator $D_{N}$, defined through the van Cittert algorithm, has the following expression : $D_{N_{i}}:=\sum_{n=0}^{N_{i}}\left(\mathrm{I}-G_{i}\right)^{n}$. We use this notation since also the deconvolution order of the two operators can be different.

Therefore, the initial value problem that we consider is :

$$
\begin{aligned}
& \partial_{t} \mathbf{w}+\nabla \cdot G_{1}\left(D_{N_{1}} \mathbf{w} \otimes D_{N_{1}} \mathbf{w}\right)-\nabla \cdot G_{1}\left(D_{N_{2}} \mathbf{b} \otimes D_{N_{2}} \mathbf{b}\right)+\nabla q-\nu \Delta \mathbf{w}=G_{1} \mathbf{f}, \\
& \partial_{t} \mathbf{b}+\nabla \cdot G_{2}\left(D_{N_{1}} \mathbf{w} \otimes D_{N_{2}} \mathbf{b}\right)-\nabla \cdot G_{2}\left(D_{N_{2}} \mathbf{b} \otimes D_{N_{1}} \mathbf{w}\right)-\mu \Delta \mathbf{b}=0, \\
& \nabla \cdot \mathbf{w}=\nabla \cdot \mathbf{b}=0, \\
& \mathbf{w}(0, \mathbf{x})=G_{1} \mathbf{u}_{0}(\mathbf{x}), \quad \mathbf{b}(0, \mathbf{x})=G_{2} \mathbf{B}_{0}(\mathbf{x}), \\
& \alpha_{1}>0, \quad \alpha_{2} \geq 0,
\end{aligned}
$$


and we will work with periodic boundary conditions. As usual, we observe that the equations (3) are not the equations (2) satisfied by $(\overline{\mathbf{u}}, \overline{\mathbf{B}})$, but we are aimed at considering (3) as an approximation of (2), hence $\mathbf{w} \simeq G_{1} \mathbf{u}$ and $\mathbf{b} \simeq G_{2} \mathbf{B}$. This is mathematically sound since, at least formally,

$$
D_{N_{i}} \rightarrow A_{i}:=\mathrm{I}-\alpha_{i}^{2} \Delta \quad \text { in the limit } N_{i} \rightarrow+\infty,
$$

and hence, again formally, (3) will become the filtered MHD equations (2).

\section{Main Results}

Existence and uniqueness issues apart, which are treated (even if without the search for estimates independent of $N_{i}$ ) for instance in [11,12], for the arbitrary deconvolution order equations (3), what is challenging is to understand whether this convergence property is true or not. One would like to show that as the approximation parameters $N_{i}$ grow, then (as recently proved for the Navier-Stokes equations in [5])

$$
\mathbf{w} \rightarrow G_{1} \mathbf{u}, \quad \mathbf{b} \rightarrow G_{2} \mathbf{B}, \quad \text { and } \quad q \rightarrow G_{1} p .
$$

Our main topic is then to study what happens when $N_{1}$ and $N_{2}$ go to infinity in (3). We prove that the sequence of models (3) converges, in some sense, to the averaged MHD equations (2), when the typical scales of filtration (called $\alpha_{1}>0, \alpha_{2} \geq 0$ ) remain fixed and the boundary conditions are the periodic ones. Before analyzing such convergence, we need to prove the existence of smooth enough solutions. To this end, we follow the same approach from [5], which revisits the approach in [9] (NSE) and now also [12] (MHD). To be more precise, let $\mathbb{T}^{3}$ be the $3 D$ torus and let $\left(\mathbf{w}_{N}, \mathbf{b}_{N}, q_{N}\right)$, with $N=\left(N_{1}, N_{2}\right)$,

$$
\begin{aligned}
& \mathbf{w}_{N}, \mathbf{b}_{N} \in \mathrm{L}^{2}\left([0, T] ; \mathrm{H}^{2}\left(\mathbb{T}_{3}\right)^{3}\right) \cap \mathrm{L}^{\infty}\left([0, T] ; \mathrm{H}^{1}\left(\mathbb{T}_{3}\right)^{3}\right), \\
& q_{N} \in \mathrm{L}^{2}\left([0, T] ; \mathrm{H}^{1}\left(\mathbb{T}_{3}\right)\right) \cap \mathrm{L}^{5 / 3}\left([0, T] ; \mathrm{W}^{2,5 / 3}\left(\mathbb{T}_{3}\right)\right)
\end{aligned}
$$

denote the solution of the ADM model (3). We are able to prove existence in such a class and our main result is the following one.

Théorème 1.1. From the sequence $\left\{\left(\mathbf{w}_{N}, \mathbf{b}_{N}, q_{N}\right)\right\}_{N \in \mathbb{N}^{2}}$, one can extract a diagonal sub-sequence (still denoted $\left.\left\{\left(\mathbf{w}_{N}, \mathbf{b}_{N}, q_{N}\right)\right\}_{N \in \mathbb{N}^{2}}\right)$ such that

$$
\begin{aligned}
& \begin{array}{l}
\mathbf{w}_{N} \longrightarrow \mathbf{w} \\
\mathbf{b}_{N} \longrightarrow \mathbf{b}
\end{array}\left\{\begin{array}{l}
\text { weakly in } \mathrm{L}^{2}\left([0, T] ; \mathrm{H}^{2}\left(\mathbb{T}_{3}\right)^{3}\right), \\
\text { weakly* in } \mathrm{L}^{\infty}\left([0, T] ; \mathrm{H}^{1}\left(\mathbb{T}_{3}\right)^{3}\right), \\
\text { strongly in } \mathrm{L}^{p}\left([0, T] ; \mathrm{H}^{1}\left(\mathbb{T}_{3}\right)^{3}\right) \quad \forall 1 \leq p<\infty,
\end{array}\right. \\
& q_{N} \longrightarrow q \quad \text { weakly in } \mathrm{L}^{2}\left([0, T] ; \mathrm{H}^{1}\left(\mathbb{T}_{3}\right)\right) \cap \mathrm{L}^{5 / 3}\left([0, T] ; \mathrm{W}^{2,5 / 3}\left(\mathbb{T}_{3}\right)\right) \text {, }
\end{aligned}
$$

when $N_{1}, N_{2} \rightarrow+\infty$, and such that the system

$$
\begin{gathered}
\partial_{t} \mathbf{w}+\nabla \cdot G_{1}\left(A_{1} \mathbf{w} \otimes A_{1} \mathbf{w}\right)-\nabla \cdot G_{1}\left(A_{2} \mathbf{b} \otimes A_{2} \mathbf{b}\right)-\nu \Delta \mathbf{w}+\nabla q=G_{1} \mathbf{f}, \\
\partial_{t} \mathbf{b}+\nabla \cdot G_{2}\left(A_{2} \mathbf{b} \otimes A_{1} \mathbf{w}\right)-\nabla \cdot G_{2}\left(A_{1} \mathbf{w} \otimes A_{2} \mathbf{b}\right)=\mu \Delta \mathbf{b}, \\
\nabla \cdot \mathbf{w}=\nabla \cdot \mathbf{b}=0 \\
\mathbf{w}(0, \mathbf{x})=G_{1} \mathbf{u}_{0}(\mathbf{x}), \quad \mathbf{b}(0, \mathbf{x})=G_{2} \mathbf{B}_{0}(\mathbf{x})
\end{gathered}
$$

holds in the distributional sense, where we recall that $A_{i}=G_{i}^{-1}=I-\alpha_{i}^{2} \Delta$.

Moreover, the following energy inequality holds :

$$
\frac{1}{2} \frac{d}{d t}\left(\left\|A_{1} \mathbf{w}\right\|^{2}+\left\|A_{2} \mathbf{b}\right\|^{2}\right)+\nu\left\|\nabla A_{1} \mathbf{w}\right\|^{2}+\mu\left\|\nabla A_{2} \mathbf{b}\right\|^{2} \leq\left\langle\mathbf{f}, A_{1} \mathbf{w}\right\rangle
$$

As a consequence of Theorem 1.1, we deduce that the field $(\mathbf{u}, \mathbf{B}, p)=\left(A_{1} \mathbf{w}, A_{2} \mathbf{b}, A_{1} q\right)$ is a dissipative (of Leray-Hopf's type) solution to the MHD equations (1).

Remarque 1.2. If one rewrites system (2) in terms of the variables $\mathbf{w}=\overline{\mathbf{u}}$ and $\mathbf{u}=A \overline{\mathbf{u}}=A \mathbf{w}$, one obtains exactly the system (4). This is not a LES model, since it is just a change of variables. The LES modeling comes into the equations with the approximation of the operators $A_{i}$ by means of the family $\left\{D_{N_{i}}\right\}_{N_{i} \in \mathbb{N}}$. 


\section{BRIEF SKETCH OF THE PROOF}

We briefly present the main ideas of the proof; for further details and extensions, and for a wider bibliography, see [3].

\subsection{Existence and uniqueness}

We use the usual Galerkin method working with divergence-free finite dimensional approximate velocities and magnetic fields. We start by constructing approximate solutions $\left(\mathbf{w}_{m}, \mathbf{b}_{m}\right)$ solving ordinary differential equations. Next, we look for bounds on $\left\{\left(\mathbf{w}_{m}, \mathbf{b}_{m}\right)\right\}_{m \in \mathbb{N}}$ and $\left\{\left(\partial_{t} \mathbf{w}_{m}, \partial_{t} \mathbf{b}_{m}\right)\right\}_{m \in \mathbb{N}}$, uniform with respect to $m \in \mathbb{N}$, by using energy-type equalities for $\left(A_{1}^{1 / 2} D_{N_{1}}^{1 / 2}\left(\mathbf{w}_{m}\right), A_{2}^{1 / 2} D_{N_{2}}^{1 / 2}\left(\mathbf{b}_{m}\right)\right)$ and the Fourier representation of the involved operators. We get

$$
\begin{aligned}
& \frac{1}{2} \frac{d}{d t}\left(\left\|A_{1}^{\frac{1}{2}} D_{N_{1}}^{\frac{1}{2}}\left(\mathbf{w}_{m}\right)\right\|^{2}+\left\|A_{2}^{\frac{1}{2}} D_{N_{2}}^{\frac{1}{2}}\left(\mathbf{b}_{m}\right)\right\|^{2}\right)+\nu\left\|\nabla A_{1}^{\frac{1}{2}} D_{N_{1}}^{\frac{1}{2}}\left(\mathbf{w}_{m}\right)\right\|^{2}+\mu\left\|\nabla A_{2}^{\frac{1}{2}} D_{N_{2}}^{\frac{1}{2}}\left(\mathbf{b}_{m}\right)\right\|^{2} \\
& \quad=\left(A_{1}^{\frac{1}{2}} D_{N_{1}}^{\frac{1}{2}}\left(G_{1} \mathbf{f}_{1 / m}\right), A_{1}^{\frac{1}{2}} D_{N_{1}}^{\frac{1}{2}}\left(\mathbf{w}_{m}\right)\right)
\end{aligned}
$$

and some a priori estimates that we organize in the table below (6). In the first column we have labeled the estimates, while the second column specifies the variable under concern. The third one explains the bound in term of function spaces. Finally, the fourth column states the order in terms of $\alpha, m$ and $N$ for each bound. Of course, each bound is of order of magnitude

$$
O\left(\left\|\mathbf{u}_{0}\right\|_{\mathrm{L}^{2}}+\left\|\mathbf{B}_{0}\right\|_{\mathrm{L}^{2}}+\frac{1}{\nu}\|\mathbf{f}\|_{\mathrm{L}^{2}\left([0, T] ; \mathrm{L}^{2}\right)}\right)
$$

\begin{tabular}{|c|c|c|c|}
\hline Label & Variable & Bound & Order \\
\hline (a) & $A_{1}^{\frac{1}{2}} D_{N_{1}}^{\frac{1}{2}}\left(\mathbf{w}_{m}\right), A_{2}^{\frac{1}{2}} D_{N_{2}}^{\frac{1}{2}}\left(\mathbf{b}_{m}\right)$ & $\mathrm{L}^{\infty}\left([0, T] ; \mathrm{H}^{0}\left(\mathbb{T}_{3}\right)^{3}\right) \cap \mathrm{L}^{2}\left([0, T] ; \mathrm{H}^{1}\left(\mathbb{T}_{3}\right)^{3}\right)$ & $O(1)$ \\
\hline (b) & $D_{N_{1}}^{1 / 2}\left(\mathbf{w}_{m}\right), D_{N_{2}}^{1 / 2}\left(\mathbf{b}_{m}\right)$ & $\mathrm{L}^{\infty}\left([0, T] ; \mathrm{H}^{0}\left(\mathbb{T}_{3}\right)^{3}\right) \cap \mathrm{L}^{2}\left([0, T] ; \mathrm{H}^{1}\left(\mathbb{T}_{3}\right)^{3}\right)$ & $O(1)$ \\
\hline$(c)$ & $D_{N_{1}}^{1 / 2}\left(\mathbf{w}_{m}\right), D_{N_{2}}^{1 / 2}\left(\mathbf{b}_{m}\right)$ & $\mathrm{L}^{\infty}\left([0, T] ; \mathrm{H}^{1}\left(\mathbb{T}_{3}\right)^{3}\right) \cap \mathrm{L}^{2}\left([0, T] ; \mathrm{H}^{2}\left(\mathbb{T}_{3}\right)^{3}\right)$ & $O\left(\alpha^{-1}\right)$ \\
\hline$(d)$ & $\mathbf{w}_{m}, \mathbf{b}_{m}$ & $\mathrm{~L}^{\infty}\left([0, T] ; \mathrm{H}^{0}\left(\mathbb{T}_{3}\right)^{3}\right) \cap \mathrm{L}^{2}\left([0, T] ; \mathrm{H}^{1}\left(\mathbb{T}_{3}\right)^{3}\right)$ & $O(1)$ \\
\hline$(e)$ & $\mathbf{w}_{m}, \mathbf{b}_{m}$ & $\mathrm{~L}^{\infty}\left([0, T] ; \mathrm{H}^{1}\left(\mathbb{T}_{3}\right)^{3}\right) \cap \mathrm{L}^{2}\left([0, T] ; \mathrm{H}^{2}\left(\mathbb{T}_{3}\right)^{3}\right)$ & $O\left(\alpha^{-1}\right)$ \\
\hline$(f)$ & $D_{N_{1}}\left(\mathbf{w}_{m}\right), D_{N_{2}}\left(\mathbf{b}_{m}\right)$ & $\mathrm{L}^{\infty}\left([0, T] ; \mathrm{H}^{0}\left(\mathbb{T}_{3}\right)^{3}\right) \cap \mathrm{L}^{2}\left([0, T] ; \mathrm{H}^{1}\left(\mathbb{T}_{3}\right)^{3}\right)$ & $O(1)$ \\
\hline$(g)$ & $D_{N_{1}}\left(\mathbf{w}_{m}\right), D_{N_{2}}\left(\mathbf{b}_{m}\right)$ & $\mathrm{L}^{\infty}\left([0, T] ; \mathrm{H}^{1}\left(\mathbb{T}_{3}\right)^{3}\right) \cap \mathrm{L}^{2}\left([0, T] ; \mathrm{H}^{2}\left(\mathbb{T}_{3}\right)^{3}\right)$ & $O\left(\frac{\sqrt{N_{i}+1}}{\alpha}\right)$ \\
\hline$(h)$ & $\partial_{t} \mathbf{w}_{m}, \partial_{t} \mathbf{b}_{m}$ & $\mathrm{~L}^{2}\left([0, T] ; \mathrm{H}^{0}\left(\mathbb{T}_{3}\right)^{3}\right)$ & $O\left(\alpha^{-1}\right)$. \\
\hline
\end{tabular}

and this is the reason why we do not mention it in the table. All bounds except (6-g) are uniform in $N$ as well, where $N \in \mathbb{N}$ is the index related to the order of deconvolution of the model.

In the previous table, $\alpha=\alpha_{1}$ for $\mathbf{w}_{m}, \alpha=\alpha_{2}$ for $\mathbf{b}_{m}$, while in (h) we can take $\alpha:=\min \left\{\alpha_{1}, \alpha_{2}\right\}$ for both $\mathbf{w}_{m}$ and $\mathbf{b}_{m}$.

Next, we use Aubin-Lions compactness result on the sequence of Galerkin approximate solutions to pass to the limit when $m \rightarrow \infty$ and $N$ is fixed and to obtain a solution to the model. Finally, we check the question of initial data and of the uniqueness of the solution.

\subsection{Convergence}

We now denote by $\left(\mathbf{w}_{N}, \mathbf{b}_{N}, q_{N}\right)$, for a given $N \in \mathbb{N}$, the unique "regular weak" solution to Problem 3, where $N=N\left(N_{1}, N_{2}\right) \rightarrow+\infty$ as $N_{1}, N_{2} \rightarrow+\infty$. We need to prove that the sequence $\left\{\left(\mathbf{w}_{N}, \mathbf{b}_{N}, q_{N}\right)\right\}_{N \in \mathbb{N}}$ has at least a sub-sequence which converges to some $(\mathbf{w}, \mathbf{b}, q)$ that is a solution to the averaged MHD equations (4).

First, we look for additional estimates, uniform in $N$, to get compactness properties about the sequences $\left\{D_{N_{1}}\left(\mathbf{w}_{N}\right), D_{N_{2}}\left(\mathbf{b}_{N}\right)\right\}_{N \in \mathbb{N}}$ and $\left\{\mathbf{w}_{N}, \mathbf{b}_{N}\right\}_{N \in \mathbb{N}} ;$ see Table 7 , where $\alpha=\min \left\{\alpha_{1}, \alpha_{2}\right\}$.

The main challenge in this process is to pass to the limit in the nonlinear terms. 


\begin{tabular}{|r|r|c|l|}
\hline Label & Variable & Bound & Order \\
\hline$(a)$ & $\mathbf{w}_{N}, \mathbf{b}_{N}$ & $\mathrm{~L}^{\infty}\left([0, T] ; \mathrm{H}^{0}\left(\mathbb{T}_{3}\right)^{3}\right) \cap \mathrm{L}^{2}\left([0, T] ; \mathrm{H}^{1}\left(\mathbb{T}_{3}\right)^{3}\right)$ & $O(1)$ \\
\hline$(b)$ & $\mathbf{w}_{N}, \mathbf{b}_{N}$ & $\mathrm{~L}^{\infty}\left([0, T] ; \mathrm{H}^{1}\left(\mathbb{T}_{3}\right)^{3}\right) \cap \mathrm{L}^{2}\left([0, T] ; \mathrm{H}^{2}\left(\mathbb{T}_{3}\right)^{3}\right)$ & $O\left(\alpha^{-1}\right)$ \\
\hline$(c)$ & $D_{N_{1}}\left(\mathbf{w}_{N}\right), D_{N_{2}}\left(\mathbf{b}_{N}\right)$ & $\mathrm{L}^{\infty}\left([0, T] ; \mathrm{H}^{0}\left(\mathbb{T}_{3}\right)^{3}\right) \cap \mathrm{L}^{2}\left([0, T] ; \mathrm{H}^{1}\left(\mathbb{T}_{3}\right)^{3}\right)$ & $O(1)$ \\
\hline$(d)$ & $\partial_{t} \mathbf{w}_{N}, \partial_{t} \mathbf{b}_{N}$ & $\mathrm{~L}^{2}\left([0, T] \times \mathbb{T}_{3}\right)^{3}$ & $O\left(\alpha^{-1}\right)$ \\
\hline$(e)$ & $q_{N}$ & $\mathrm{~L}^{2}\left([0, T] ; \mathrm{H}^{1}\left(\mathbb{T}_{3}\right)\right) \cap \mathrm{L}^{5 / 3}\left([0, T] ; \mathrm{W}^{2,5 / 3}\left(\mathbb{T}_{3}\right)\right)$ & $O\left(\alpha^{-1}\right)$ \\
\hline$(f)$ & $\partial_{t} D_{N_{1}}\left(\mathbf{w}_{N}\right), \partial_{t} D_{N_{2}}\left(\mathbf{b}_{N}\right)$ & $\mathrm{L}^{4 / 3}\left([0, T] ; \mathrm{H}^{-1}\left(\mathbb{T}_{3}\right)^{3}\right)$ & $O(1)$ \\
\hline
\end{tabular}

We can now pass to the limit. From the above estimates and classical rules of functional analysis, we can infer that there exist

$$
\begin{aligned}
& \mathbf{w}, \mathbf{b} \in \mathrm{L}^{\infty}\left([0, T] ; \mathrm{H}^{1}\left(\mathbb{T}_{3}\right)^{3}\right) \cap \mathrm{L}^{2}\left([0, T] ; \mathrm{H}^{2}\left(\mathbb{T}_{3}\right)^{3}\right), \\
& \mathbf{z}_{1}, \mathbf{z}_{2} \in \mathrm{L}^{\infty}\left([0, T] ; \mathrm{H}^{0}\left(\mathbb{T}_{3}\right)^{3}\right) \cap \mathrm{L}^{2}\left([0, T] ; \mathrm{H}^{1}\left(\mathbb{T}_{3}\right)^{3}\right), \\
& q \in \mathrm{L}^{2}\left([0, T] ; \mathrm{H}^{1}\left(\mathbb{T}_{3}\right)\right) \cap \mathrm{L}^{5 / 3}\left([0, T] ; \mathrm{W}^{2,5 / 3}\left(\mathbb{T}_{3}\right)\right),
\end{aligned}
$$

such that, up to sub-sequences,

$$
\begin{aligned}
& \begin{array}{l}
\mathbf{w}_{N} \longrightarrow \mathbf{w} \\
\mathbf{b}_{N} \longrightarrow \mathbf{b}
\end{array} \\
& \begin{array}{l}
\partial_{t} \mathbf{w}_{N} \longrightarrow \partial_{t} \mathbf{w} \\
\partial_{t} \mathbf{b}_{N} \longrightarrow \partial_{t} \mathbf{b}
\end{array} \\
& \begin{array}{l}
D_{N_{1}}\left(\mathbf{w}_{N}\right) \longrightarrow \mathbf{z}_{1} \\
D_{N_{2}}\left(\mathbf{b}_{N}\right) \longrightarrow \mathbf{z}_{2} \\
\partial_{t} D_{N_{1}}\left(\mathbf{w}_{N}\right) \longrightarrow \partial_{t} \mathbf{z}_{1} \\
\partial_{t} D_{N_{2}}\left(\mathbf{b}_{N}\right) \longrightarrow \partial_{t} \mathbf{z}_{2} \\
\text { weakly* in } \mathrm{L}^{\infty}\left([0, T] ; \mathrm{H}^{1}\left(\mathbb{T}_{3}\right)^{3}\right), \\
\text { strongly in } \mathrm{L}^{p}\left([0, T] ; \mathrm{H}^{1}\left(\mathbb{T}_{3}\right)^{3}\right) \forall 1 \leq p<\infty,
\end{array} \\
& \begin{array}{l}
\text { weakly in } \mathrm{L}^{2}\left([0, T] \times \mathbb{T}_{3}\right)^{3}, \\
q_{N} \longrightarrow q
\end{array}
\end{aligned} \quad \begin{aligned}
& \text { weakly in } \mathrm{L}^{2}\left([0, T] ; \mathrm{H}^{1}\left(\mathbb{T}_{3}\right)^{3}\right), \\
& \text { weakly* in } \mathrm{L}^{\infty}\left([0, T] ; \mathrm{H}^{0}\left(\mathbb{T}_{3}\right)^{3}\right), \\
& \text { strongly in } \mathrm{L}^{p}\left([0, T] \times \mathbb{T}_{3}\right)^{3} \forall 1 \leq p<10 / 3,
\end{aligned}
$$

We notice that

$$
\begin{array}{lll}
D_{N_{1}} \mathbf{w}_{N} \otimes D_{N_{1}} \mathbf{w}_{N} \longrightarrow \mathbf{z}_{1} \otimes \mathbf{z}_{1} & \text { strongly in } \mathrm{L}^{p}\left([0, T] \times \mathbb{T}_{3}\right)^{9}, & \forall 1 \leq p<5 / 3, \\
D_{N_{2}} \mathbf{b}_{N} \otimes D_{N_{2}} \mathbf{b}_{N} \longrightarrow \mathbf{z}_{2} \otimes \mathbf{z}_{2} & \text { strongly in } \mathrm{L}^{p}\left([0, T] \times \mathbb{T}_{3}\right)^{9}, & \forall 1 \leq p<5 / 3, \\
D_{N_{1}} \mathbf{w}_{N} \otimes D_{N_{2}} \mathbf{b}_{N} \longrightarrow \mathbf{z}_{1} \otimes \mathbf{z}_{2} & \text { strongly in } \mathrm{L}^{p}\left([0, T] \times \mathbb{T}_{3}\right)^{9}, & \forall 1 \leq p<5 / 3,
\end{array}
$$

while all other terms in the equation pass easily to the limit as well. Our proof will be complete as soon as we will have checked that $\mathbf{z}_{1}=A_{1} \mathbf{w}$ and $\mathbf{z}_{2}=A_{2} \mathbf{b}$.

We prove just the first one being the other completely similar. Let us consider a vector field $\mathbf{v} \in \mathrm{L}^{2}\left([0, T] ; \mathrm{H}^{2}\left(\mathbb{T}_{3}\right)^{3}\right) ;$ we have $\left(D_{N_{1}}\left(\mathbf{w}_{N}\right), \mathbf{v}\right)=\left(\mathbf{w}_{N}, D_{N_{1}}(\mathbf{v})\right)$. We claim that $D_{N_{1}}(\mathbf{v}) \rightarrow A_{1} \mathbf{v}$ 
strongly in $\mathrm{L}^{2}\left([0, T] \times \mathbb{T}_{3}\right)^{3}$. If such a convergence result holds, we have

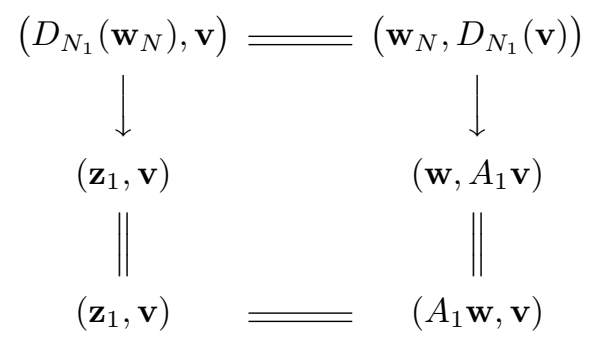

yielding $\mathbf{z}_{1}=A_{1} \mathbf{w}$. The convergence of $D_{N_{1}}$ to $A_{1}$ follows by a direct calculation with Fourier series expansion.

To conclude, we prove that the solution $(\mathbf{w}, \mathbf{b})$ satisfies an energy inequality. Observe that, from the previous estimates, we obtain (as a consequence of the lower bound on the operator $D_{N}$ ) that

$$
\begin{aligned}
& \mathbf{w}_{N}, \mathbf{b}_{N} \in \mathrm{L}^{2}\left([0, T] ; \mathrm{H}^{2}\left(\mathbb{T}_{3}\right)^{3}\right) \quad \text { uniformly in } N \\
& \mathbf{w}_{N}, \mathbf{b}_{N} \in \mathrm{L}^{\infty}\left([0, T] ; \mathrm{H}^{2}\left(\mathbb{T}_{3}\right)^{3}\right) \quad \text { NON uniformly in } N ;
\end{aligned}
$$

hence, obtaining an estimate for $\mathbf{w}, \mathbf{b}$ in $\mathrm{L}^{\infty}\left(0, T ; \mathrm{H}^{2}\left(\mathbb{T}_{3}\right)^{3}\right)$ is not trivial at all since it does not derive directly from the various estimates collected in the tables.

Proposition 2.1. The solution $(\mathbf{w}, \mathbf{b}, q)$ satisfies the energy inequality

$$
\frac{1}{2} \frac{d}{d t}\left(\left\|A_{1} \mathbf{w}\right\|^{2}+\left\|A_{2} \mathbf{b}\right\|^{2}\right)+\nu\left\|\nabla A_{1} \mathbf{w}\right\|^{2}+\mu\left\|\nabla A_{2} \mathbf{b}\right\|^{2} \leq\left(\mathbf{f}, A_{1} \mathbf{w}\right)
$$

in the sense of distributions. This implies that $(\mathbf{w}, \mathbf{b})$ is the average of a weak (in the sense of Leray-Hopf) or dissipative solution $(\mathbf{u}, \mathbf{B})$ of the MHD equations (1). In fact, the energy inequality can also be read as

$$
\frac{1}{2} \frac{d}{d t}\left(\|\mathbf{u}\|^{2}+\|\mathbf{B}\|^{2}\right)+\nu\left\|\nabla A_{1} \mathbf{u}\right\|^{2}+\mu\left\|\nabla A_{2} \mathbf{b}\right\|^{2} \leq(\mathbf{f}, \mathbf{u}) .
$$

If we assume less regularity on the external force, as for instance $\mathbf{f} \in L^{2}\left([0, T] ; \mathrm{H}^{-1}\left(\mathbb{T}_{3}\right)^{3}\right)$, the proof remains the same and we obtain the corresponding inequality

$$
\frac{1}{2} \frac{d}{d t}\left(\left\|A_{1} \mathbf{w}\right\|^{2}+\left\|A_{2} \mathbf{b}\right\|^{2}\right)+\nu\left\|\nabla A_{1} \mathbf{w}\right\|^{2}+\mu\left\|\nabla A_{2} \mathbf{b}\right\|^{2} \leq\left\langle\mathbf{f}, A_{1} \mathbf{w}\right\rangle .
$$

\section{REFERENCES}

[1] N. A. Adams and S. Stolz. Deconvolution methods for subgrid-scale approximation in large eddy simulation. Modern Simulation Strategies for Turbulent Flow, R.T. Edwards, 2001.

$[2]$ L. C. Berselli. Towards fluid equations by approximate deconvolution models. In Partial differential equations and fluid mechanics, London Math. Soc. Lecture Note Ser., pages?+21. Cambridge Univ. Press, Cambridge, 2012. In press.

[3] L. C. Berselli, D. Catania, and R. Lewandowski. Convergence of approximate deconvolution models to the mean Magnetohydrodynamics Equations : Analysis of two models. Preprint arXiv :1206.1483v1.

[4] L. C. Berselli, T. Iliescu, and W. J. Layton. Mathematics of Large Eddy Simulation of turbulent flows. Scientific Computation. Springer-Verlag, Berlin, 2006.

[5] L. C. Berselli and R. Lewandowski. Convergence of approximate deconvolution models to the mean NavierStokes equations. Ann. Inst. H. Poincaré (C), Non Linear Analysis, 29 :171-198, 2012.

[6] D Catania. Length-scale estimates for the 3D simplified Bardina magnetohydrodynamic model. J. Math. Phys., $52(5): 053101,1-13,2011$

[7] D. Catania and P. Secchi. Global existence for two regularized MHD models in three space-dimension. Port. Math., 68(1) :41-52, 2011

[8] P. A. Davidson. An introduction to magnetohydrodynamics. Cambridge Texts in Applied Mathematics. Cambridge University Press, Cambridge, 2001.

[9] A. Dunca and Y. Epshteyn. On the Stolz-Adams deconvolution model for the large-eddy simulation of turbulent flows. SIAM J. Math. Anal., 37(6) :1890-1902 (electronic), 2006.

[10] M. Holst, E. Lunasin, and G. Tsogtgerel. Analysis of a general family of regularized Navier-Stokes and MHD models. J. Nonlinear Sci., 20(5) :523-567, 2010. 
[11] A. Labovsky and C. Trenchea. Approximate deconvolution models for magnetohydrodynamics. Numer. Funct. Anal. Optim., 31(12) :1362-1385, 2010.

[12] A. Labovsky and C. Trenchea. Large eddy simulation for turbulent magnetohydrodynamic flows. J. Math. Anal. Appl., 377(2) :516-533, 2011.

[13] W. Layton, M. Sussman, and C. Trenchea. Bounds on energy, magnetic helicity, and cross helicity dissipation rates of approximate deconvolution models of turbulence for MHD flows. Numer. Funct. Anal. Optim., 31(46) :577-595, 2010.

[14] W. J. Layton and L. Rebholz. Approximate Deconvolution Models of Turbulence, volume 2042 of Lecture Notes in Mathematics. Springer, Heidelberg, 2012.

[15] R. Lewandowski. On a continuous deconvolution equation for turbulence models. Lecture Notes of Neças Center for Mathematical Modeling, $5: 62-102,2009$.

[16] J. S. Linshiz and E. S. Titi. Analytical study of certain magnetohydrodynamic- $\alpha$ models. J. Math. Phys., $48(6): 065504,28,2007$.

[17] S. Stolz and N. A. Adams. An approximate deconvolution procedure for large-eddy simulation. Phys. Fluids, 11(7) :1699-1701, 1999.

[18] S. Stolz, N. A. Adams, and L. Kleiser. An approximate deconvolution model for large-eddy simulation with application to incompressible wall-bounded flows. Phys. Fluids, 13(4) :997-1015, 2001. 